\title{
TETANUS.
}

By RONALD W. RAVEN, F.R.C.S.

(Surgeon E.M.S.; Surgeon to Out-Patients, French Hospital, London; Assistant Surgeon Royal Cancer Hospital (Free); Assistant Surgeon, Gordon Hospital.)

The incidence of tetanus varies widely in various theatres of warfare. During the Peninsular Wars hundreds of our soldiers died as the result of tetanus but during the Crimean War and the American War of Independence the incidence was low, namely 0.2 per cent. Tetanus was not seen during the Nile Expedition, South African War and Russo-Japanese War. During the Franco-German War the incidence was 0.35 per cent. and it was noticed that the numbers of cases with the disease varied according to the spheres of operations. Experiences in the Great War I9I4-I9I8 showed that the incidence of tetanus was insignificant outside French and Belgian territory. The incidence was high in the northern districts of France.

\section{r. Bacteriology.}

\section{Pathological Features.}

The cause of tetanus in man and animals is the Bacillus Tetani, first adequately described by Rosenbach. This organism belongs to a species the members of which have similar characters ; the others, however, do not possess the power of producing tetanus. The species is composed of anaerobic organisms widely distributed in soil and the intestinal tract. Their presence in soil is due to admixture with fæces of horses and cattle. An outstanding characteristic is their capacity for developing a spherical spore at the end of the rod especially when cultured in a "digest" medium. In the species the important organism is the tetanus-producing strains of Bacillus Tetani.

In the non-sporing condition B.tetani shows no characteristic feature and is seen to be a bacillus with slightly rounded ends varying in width from 0.6 to $0.3 \mu$. It varies also in length from several $\mu$ to over two diameters of the field. The sporing phase of B.tetani is characterised by "drumstick" forms. At first there is a slight oval enlargement of one end of the bacillus which does not retain carbol-fuchsin; later it acquires a spherical shape and stains deeply. The organism is actively motile due to the presence of flagella described by Kanthack and Connell Certain non-motile varieties have also been isolated. With regard to its staining reactions the body stains with any of the basic aniline dyes and the ripened spore stains with carbol-fuchsin. The organism is gram-positive. . Strict anaerobic conditions are necessary for its cultivation or a certain degree of reducing intensity in the medium such as cotton wool or meat particles.

2. The genesis of tetanus.

Much experimental work has been carried out concerning the absorption and mode of action of tetanus toxin. The recent experimental work is published in the Bull. Johns Hopk. Hosp., Vols 56 and 57. There are two hypotheses regarding the mechanism of absorption of tetanus toxin and its mode of action on tissue cells and in the present state of our knowledge it is impossible to state which is correct.

1. The toxin is absorbed by the motor nerve endings and is carried along the axis cylinders to the anterior horn cells of the central nervous system. These cells are stimulated and produce muscular contractions. Later the toxin diffuses through the spinal cord and horn muscles in other parts of the body become affected.

2. The toxin is absorbed by the lymphatic vessels, carried to the general circulatory system and distributed to the central nervous system by the arteries. 
3. The incubation period.

An incubation period always intervenes between infection and the onset of symptoms. This period varies with the site of innoculation and it may be stated that the more peripheral the infection, the longer is the incubation period. The usual mean incubation period is about nine days; the shortest period recorded is eighteen hours and delayed tetanus has been observed as long as two and a half years after infection. Experimental work has shown the differences in the incubation period with the tissue innoculated. Thus after injection into nerves, cord or brain the period becomes progressively shorter, to a minimum of one hour after intracerebral injection in the guinea pig. The period is longest after intravenous injection and is longer after subcutaneous than after intramuscular injection. A prophylactic injection of antitoxin considerably lengthens the incubation period.

\section{Clinical Features.}

I. Classification of tetanus according to type of disease.

There are four main types of tetanus which were classified by the Tetanus Committee established in I9I6. The following table correlates the type of disease with the prognosis (after Bruce, D.).

TABLE I.

Tetanus-type of disease correlated with prognosis.

Type of Disease.

Total No. of Cases. Recovered.

Died.

Mortality.

1. Trismus the earliest symptoms:

(a) With complete closure of jaws developing within 24 hours of the onset of symptoms

(b) With complete closure of jaws developing after 24 hours of the onset

... $\quad \ldots$

(c) With incomplete closure of jaws

\begin{tabular}{rrrrrrr}
77 & $\ldots$ & 22 & $\ldots$ & 55 & $\ldots$ & 71.4 p.c. \\
& & & & & & \\
80 & $\ldots$ & 40 & $\ldots$ & 40 & $\ldots$ & 50.0 p.c. \\
505 & $\ldots$ & 380 & $\ldots$ & 125 & $\ldots$ & 24.9 p.c. \\
& & & & & & \\
134 & $\ldots$ & 109 & $\ldots$ & 25 & $\ldots$ & 18.6 p.c. \\
50 & $\ldots$ & 38 & $\ldots$ & 12 & $\ldots$ & 24.0 p.c. \\
201 & $\ldots$ & 201 & $\ldots$ & - & $\ldots$ & 0.0 p.c. \\
\hline 1,047 & $\ldots$ & 790 & $\ldots$ & 257 & $\ldots$ & 24.5 p.c. \\
\hline
\end{tabular}

2. Trismus occurring after other symptoms of tetanus have shown $\begin{array}{llll}\text { themselves } & \ldots & \ldots & \ldots\end{array}$

3. General tetanus without trismus

4. Local tetanus

Total ... $\quad .$.

1,047

The figures given in Table I illustrate the importance of classifying cases with tetanus as this forms a valuable basis for assessing prognosis.

2. The type of wound and the incidence of tetanus.

In an analysis of I57 cases in order to determine whether the mortality from tetanus is greater with wounds of the body than of the limbs, Leishman and Smallman found that a fatal issue is more common with wounds of the body. Thus 53 per cent. of patients with wounds of the body died as opposed to 47 per cent. with wounds of the limbs. These observers also investigated the relationship between the mortality from tetanus and the condition of the wound from the point of view of sepsis, presence of gas gangrene, severity of the damage and the presence of multiple wounds. The figures produced show the importance of these factors in fatal cases of tetanus. The frequency of gas gangrene is much greater as a complicating factor in the cases which terminated fatally. 
It has been stated that the presence of a fracture increases the mortality rate but the figures given by Bruce show that the mortality from tetanus in wounds complicated by a fracture was 29.I per cent. and in wounds with no fracture it was 37.9 per cent.

Tetanus may supervene after a surgical operation at the site of a wound and therefore, it is advisable to administer a prophylactic injection of serum before the operation is undertaken even if the wound is healed. It has been proved that the tetanus bacillus may be harboured for many years in healed wounds.

\section{Symptomatolgy.}

A. Classical tetanus.

In the successful treatment of tetanus it is of paramount importance to recognise the disease in its earliest phase. The symptomatology will therefore be considered under two headings-early and late.

I. Early symptomatology.

In the early phase the patient develops an anxious expression, suffers from insomnia, distressing dreams or delirium. He may develop a violent headache and exhibit bouts of temper. Profuse local or generalised sweating is present. There is pain in the neck and back, tremor when the tongue is protruded, soreness of the throat and excessive yawning. Difficulty in micturition may be present due to spasm of the sphincter of the bladder. Spasm or stiffness of the injured limbs may be noticed and attention should be given to any hardness of the muscles in the vicinity of the wound. Later other symptoms manifest themselves, such as dysphagia, increased salivation, nystagmus, strabismus, spasmodic cough and a severe stitch in the side. From the onset of the disease all reflexes, both superficial and deep, are increased and Babinski's sign is positive.

\section{Late symptomatology.}

The most important symptom is trismus due to spasm of the masseter muscles. There may also be stiffness and pain at the back of the neck. Other groups of muscles are later involved, including the musculature of the anterior abdominal wall, of the back and lower extremities. The musculature of the forearms and hands tends to escape. The face assumes the typical risus sardonicus expression, the eyebrows being raised and the angles of the mouth drawn out. Marked dysphagia is often present.

As the disease progresses paroxysmal spasms of muscles occur and the patient may assume the position of opisthotonos. Positions of pleurothotonos and emprosthotonos are less frequently seen. These paroxysmal spasms cause intense suffering, muscles may be ruptured and fractures of bones occur. Owing to spasm of the intercostal muscles and diaphragm, breathing is hampered and cyanosis develops. Over-action of sphincters results in retention of urine and constipation.

Fever is rarely present: any increased temperature may be accounted for by the condition of the wound. High temperatures have been recorded just before death. The pulse-rate is always increased.

The mind of the patient remains clear until near the end; the patient is fully aware of his serious condition.

B. Atypical Forms of tetanus.

Atypical forms of tetanus occur as the result of the use of prophylactic antitetanic serum and they may be classified according to Courtois Suffit and Giroux 
in three main groups, namely: (I) Splanchnic tetanus; (2) Cephalic tetanus: (3) Local tetanus of the limbs. In this variety of the disease there is an absence of general spasms with opisthotonos.

I. Splanchnic tetanus.

This is a very fatal form and complicates visceral injury in penetrating wounds of the abdomen and chest. The outstanding features are severe dysphagia and acute dyspnœa due to spasm of the muscles of deglutition and respiration.

\section{Cephalic tetanus.}

There are four varieties of cephalic tetanus, namely: (a) non-paralytic; (b) with facial paralysis; (c) with oculo-motor paralysis; (d) with hypoglossal paralysis. The non-paralytic variety is the least common and the hypoglossal variety is not often seen.

Cephalic tetanus with facial paralysis follows a wound of the face or neighbouring structures and is characterised by unilateral trismus which may become bilateral; dysphagia and facial paralysis on the same side as the wound. Spasms occur in the muscles on the paralysed side of the face.

Cephalic tetanus with oculo-motor paralysis complicates wounds of the orbit or contiguous structures. Ptosis is the commonest sign and any, or all, of the ocular muscles may be paralysed.

3. Local tetanus of the limbs.

Tetanus may be localised to one arm, one leg or both legs. These have been described as the monoplegic and paraplegic types. The chief symptoms are clonic and tonic spasms occurring in the wounded limbs and there may be considerable pain. Burrows, $H$. has called attention to a special reflex which is indicative of tetanus. This reflex consists of extension of the foot and leg when the sole is stimulated; the normal reflex being withdrawal of the foot from the stimulus.

\section{Prophylactic treatment.}

\section{Treatment.}

(a) The use of tetanus antitoxin.

This is the most important aspect of the treatment of tetanus for the disease can be prevented by active immunisation against it. In the Great War all belligerents endeavoured to give a prophylactic dose of antitoxin to every wounded man. In the British Army 500 U.S.A. units of antitoxin were given and a study of the statistics concerning the incidence of tetanus shows the beneficial effects produced. The figures set out in Table II were compiled by Bruce, D.

TABLE II.

Tetanus-effect of prophylaxis on mortality.

Type of Patient.

$\begin{array}{lllllll}\text { Protected } & \ldots & \ldots & \ldots & \ldots & \ldots & 22 \\ \text { per cent. }\end{array}$

Unprotected and type not stated ... 53 , ,

Tetanus in " trench feet"-effect of prophylaxis on mortality.

Protected

Unprotected
16 per cent.

75 
Recent work has shown that following the administration of $I, 500$ units of tetanus antitoxin the recipient's serum contains 0.1 to 0.25 units of antitoxin per c.c. During the following 4 to II days this titre is maintained and then begins to diminish. Therefore in cases with extensive injuries, or injuries requiring operative interference at a time longer than II days of the injury, a second dose of antitoxin should be given. The required dose bears no relation to the age of the patient. In certain cases it has been found that a prophylactic injection has failed to protect the patient from the disease but the symptoms have been ameliorated and the mortality lowered.

(b) The use of tetanus toxoid.

On account of the effects produced by tetanus antitoxin, such as serum sickness, a substitute substance in tetanus toxoid has been produced. The French immunologists have done a considerable amount of experimental work with this substance and the substance has been used very extensively in the French Army.

An important principle to remember in active immunization by tetanus toxoid is that after three subcutaneous injections at definite intervals, a fourth injection will rapidly raise the titre of antitoxin in the patient's serum which will protect him from tetanus. This response to the fourth injection can be evoked at least five years after the immunizing doses. Further, the response to the fourth injection is so rapid as to obviate the administration of antitoxin.

In order to prevent allergic reactions it is advisable to inject purified alumprecipitated tetanus toxoid. The dosage is I.o c.c. given by subcutaneous injection on three occasions at intervals of from six to twelve weeks. According to Firor, two weeks after the second injection nearly all individuals show a concentration of antitoxin in the blood which is supposed to afford adequate protection. The third injection raises the titre of antitoxin in the serum above the protection level. After the injection of toxoid there is slight induration at the site of innoculation but no other undesirable effects are seen.

\section{x. Nursing care.}

\section{General Treatment.}

In the treatment of tetanus the most careful nursing of the patient is of prime importance. The patient should be placed in a well ventilated, darkened room, the walls of which should be painted dark. He must be kept at absolute rest and all noise stimuli should be eliminated. A water bed for the patient is an asset and a cradle should be used to keep the bed clothes away from the body. The wounded part must be kept at rest. A daily enema is necessary to empty the bowel as constipation is usually present. Catheterisation of the bladder is seldom required.

\section{Feeding.}

A special nurse is required by day and night to feed the patient. The patient's dietary containing at least 2,000 calories per day may be made up of glucoselemonade, eggs and milk. The fluid balance of the body must be maintained by the administration of saline, if necessary, by the rectal or other routes. If there is marked dysphagia nutrient enemata may be given and fluids must be introduced into the body by the rectal, subcutaneous or intravenous routes. Nasal feeding has been advocated but it is wise to remember that the passage of a catheter through the nose into the stomach has produced a fatal spasm. 


\section{The use of drugs.}

Narcotics are indispensable in the treatment of tetanus. Subcutaneous injections of morphia gr. $\frac{1}{6}$ several times a day and chloral hydrate gr. 30 by mouth several times a day are of value. Too much morphine should be avoided especially in the presence of pulmonary complications. If there is dysphagia, chloral hydrate -3 grm. with 50 c.c. water and 5'o c.c. mucil. amygd. may be given per rectum. Other narcotics which may be used are luminal sodium up to gr. 30 daily, or I to 2 c.c. of 20 per cent. solution in water subcutaneously; potassium bromide gr. 45 daily. Reflex spasms may be controlled by avertin given per rectum, dosage o.I c.c. per kg. of body weight. This may be repeated at intervals of 6 to 8 hours. If the respirations become rapid and shallow and if there is cyanosis a hypodermic injection of atropine sulphate gr. I/ I20 should be given.

\section{The use of Oxygen therapy.}

Oxygen may be used in the treatment of tetanus in the following ways :

(a) In the treatment of respiratory distress.

Oxygen therapy should be instituted if the respirations become rapid and shailow and cyanosis develops. In these cases warmed oxygen is administered through a nasal catheter.

(b) In the treatment of the wound.

Experiments carried out with guinea pigs have tended to show that subcutaneous injections of oxygen into a wound may delay or prevent the onset of tetanus. It is certain that the presence of oxygen in the wound will prevent the multiplication of the organisms. It is rational, therefore, to advise the local use of oxygen in deep wounds in the early phase of the disease.

\section{The use of anæsthesia.}

Attention has already been called to the value of avertin given by the rectum in the treatment of reflex spasms. It may be that severe respiratory spasms supervene before the patient is under the influence of avertin and in these cases they may be controlled by the administration of nitrous oxide gas and oxygen. Many authorities have advocated chloroform anæsthesia in the treatment of the severe spasms of tetanus but this treatment should be adopted with caution. In a number of cases fatal pulmonary் œdema has supervened and it may be that chloroform anæsthesia contributed to the development of this fatal complication.

\section{Antitoxin Treatment.}

Successful prophylactic treatment in tetanus is to prevent toxin combining with nerve cells. It has been shown that when toxin reaches the anterior horn cells it becomes fixed and irrecoverable. The problem of treatment in this stage of the disease is to discover the best means of preventing fresh supplies of toxin reaching and irreparably damaging the nerve cells. Six routes are available for the introduction of antitetanic serum into the body, namely:-(a) intracerebral; (b) intraneural; (c) subcutaneous; (d) intramuscular; (e) intravenous; (f) intrathecal, either in the lumbar region or into the cisterna magna.

The intracerebral and intraneural routes are unsuitable for general use; they have been used in the treatment of desperate cases. Regarding the other routes, 
there has been considerable controversy regarding the efficacy of each method. The administration of antitetanic serum by the intrathecal route was advised consistently by the War Office Committee for the Study of Tetanus. This view was also held by Andrewes, F. W., who in collaboration with Horder, described the successful treatment of a case in which the intravenous route was supplemented by the intrathecal route. Experiments were carried out by Sherrington in the treatment of tetanus in animals with the administration of antitetanic serum by the intrathecal route. This observer called attention to the value of this method of treatment. Golla's experiments demonstrated that good results may be obtained by the administration of serum by the combined intrathecal and intramuscular routes. As the result of the study of a series of cases, Leishman and Smallman have advised the intramuscular route. The intravenous route has found strong advocates in Dean and Cole, L. The latter observer advises a single intravenous dose of 200,000 international units of antitetanic serum and in the treatment of a series of 40 cases there was a mortality rate of 47.5 per cent. Important clinical research has been carried out by Yodh and his results obtained by each method of administration of various doses of antitoxin are set out in Table III.

TABLE III.

Effect of route and dose of serum injections in human patients suffering from tetanus. (Yodh 1932).

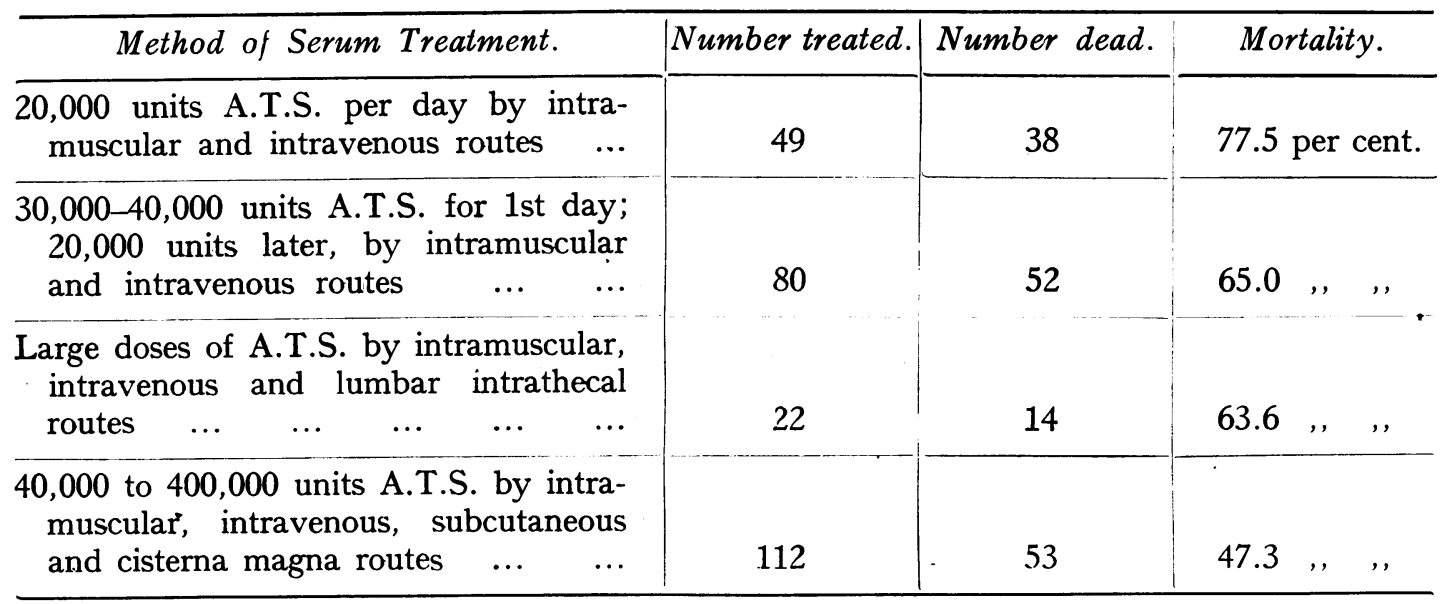

The figures set out in Table III show that large doses of A.T.S. given by the combined intramuscular, intravenous, subcutaneous and intrathecal cisterna magna routes produced the best results. Yodh states that the administration of the antitoxin into the cisterna magna is preferable to lumbar puncture.

Consideration of the evidence has led to the conclusion that it is advisable to administer large doses of antitetanic serum by the intrathecal, intravenous, intramuscular and subcutaneous routes. On the first day 15,000 U.S.A. units should be injected by each method. On each subsequent day this should be repeated except that the intravenous dose may be omitted. When improvement occurs the amount of serum given may be gradually reduced and the intrathecal route of administration omitted. Serum should be given by the intramuscular and subcutaneous routes until all reflex spasms have ceased. There is evidence that intrathecal injection into the cisterna magna is preferable to the lumbar region. 


\section{Surgical Treatment.}

In the presence of established tetanus severe operations, such as amputations, should be postponed until there are signs of improvement in the general symptomatology and some degree of artificial immunisation has occurred following the injection of adequate amounts of antitoxin. The treatment of wounds is important but this should not be undertaken hastily. Operation aims at the excision of necrotic tissues, removal of foreign bodies, and provision of free drainage. When performing an operation for excision of necrotic tissues great care must be taken to avoid opening up non-infected tissues, otherwise more toxin may be absorbed. It is also advisable to irrigate the tissues with antitoxin. The wounded part must be carefully immobilised and kept at rest until healing has occurred. Surgical treatment may also be required if there is marked laryngeal spasm leading to suffocation. In these cases tracheotomy may be necessary.

\section{Conclusion.}

A survey has been made of the problems connected with tetanus with due regard to the experiences of others in the Great War and taking into account more recent work on the subject. The pathology of the disease is considered; the symptomatology in all its phases is described; the treatment is discussed in detail and instructions are given concerning the management of a case of tetanus.

\section{REFERENCES.}

ANDREWES, F. 'W., " On the intrathecal route for the administration of tetanus antitoxin." Lancet (1917), 1, 682.

ANDREWES, F. W., and HORDER, "A case of tetanus." Lancet, (1917), 1, 685.

BRUCE, DAVID, “Tetanus.” Jour. Hygiene (1920-21), 19, 1.

BURROWS, H " Modified tetanus." Lancet (1917) 1, 139

COLE, "The treatment and prognosis of tetanus." "Proc. Roy. Soc. Med. (1938), 31, 1205.

COLE, L., "The treatment and prognosis of tetanus." Proc. Roy. Soc. Med. (1938),

DEAN, H. R., "A report on twenty-five cases of tetanus." Lancet (1917), 1, 673.

FIROR, W. M., "The prevention and treatment of tetanus." Am. Journ. Surg. (Dec. 1939), 450. 108,941 .

GOLLA, F., "A comparison of subcutaneous with intravenous and intrathecal administration of tetanus antitox in in experimental tetanus." Lancet (1917), 1, 686.

LEISHMAN, W. B. and SMALLMAN, A. B., "An analysis of recent cases of tetanus in the British Expeditionary Force." Lancet (1917), 1, 131.

SHERRINGTON C , "Öbservations with antitetanus serum in the monkey." Lancet (1917), 2,964.

SUFFIT COURTOIS and GIROUX, "Les forme anormales du tétanos." Précis de Médecine et de Chirurgie du Guerre. Paris. Masson et Cie (1916).

YODH, B. B., "Observations on the treatment of tetanus." Brit. Med. Jour. (1932), $2,589$.

War Office Committee on the study of tetanus. "Memorandum on tetanus." Brit. Med. Jour. (1916), 2, 647. 